\title{
Magnetic Resonance Image Artifact Due to the Examination Method and Scanning Sequences
}

\author{
Jozef Živčák ${ }^{1 *}$, Marianna Trebuňová ${ }^{2}$, Andrej Repovský ${ }^{3}$, Galina Laputková ${ }^{2}$ \\ 'Department of Biomedical Engineering and Measurement DBEaM, Faculty of Mechanical Engineeering, Institute of Technical Sciences, TUKE, \\ Letná 9, 04200 Košice, Slovak Republic \\ ${ }^{2}$ Department of Medical and Clinical Biophysics, Pavol Jozef Šafárik University, Faculty of Medicine, Trieda SNP 1, 04011 Košice, Slovak \\ Republic \\ ${ }^{3}$ Department of Radiology, A. Leňa Hospital, Nemocničná 7, 06601 Humenné, Slovak Republic
}

\section{BIOGRAPHICAL NOTES}

Jozef Živčák, Dr.h.c. prof. Ing. PhD., he is a graduate of Faculty of Mechanical Engineering, Technical University in Kosice (TUKE). Currently works as a specialized assistant at Department of Power Engineering. He actively participates in research of heat transfer, fluid flow, new technologies in area of renewable energy resources and mathematical modelling of energy processes.

Trebuňová Marianna, RNDr., PhD., 1979, assistant professor, graduated as Magister disciplines biology, physics, education and psychology at Faculty of science, UPJŠ Košice in 2003, RNDr., PhD. - from medical biology and molecular genetics at Faculty of medicine, UPJŠ Košice in 2008, specializes in medical biology and molecular genetics and education of biophysics.

Repovský Andrej, MUDr., 1986, medical doctor, graduated in general medicine at Faculty of medicine, UPJŠ Košice in 2010, nowadays - medical doctor at Department of radiology in A.Leňa Hospital, Humenné.

Laputková Galina, RNDr., PhD., 1958, researcher, graduated in applied biophysics at Kharkov State University, Faculty of Radiophysics in 1982; RNDr. - Department of Biophysics and Chemical Physics, Faculty of Mathematics and Physics, Komensky University, PhD. - Department of Biophysics and Chemical Physics, Faculty of Mathematics and Physics, Komensky University, in 1989; specializes in the study of lipid bilayer membranes, electrochemical impedance spectroscopy, cyclic voltammetry, 2D electrophoresis, and the sample preparation for MS.

\section{KEYWORDS}

Artifacts, magnetic resonance, sequences.

\section{ABSTRACT}

Magnetic resonance (MR) belongs to the most important diagnostic and imaging methods. It is derived from classic nuclear magnetic resonance that is the method based upon the difference between the magnetic properties of the diverse nuclei. However, this method, as well as the other non-invasive imaging methods, is burdened by the presence of the numerous artifacts. In addition to the artifacts due to the patient, the artifacts due to the method and the scanning sequences exist. In this review the main types of MR artifacts due to the examination method and the scanning sequences are presented. The characteristic properties and the physical and the 
technical preconditions of given artifacts are given.

\section{Introduction}

The term artifact is derived from Latin words artis (art) and facere (do). This undesirable "art" is concerning to the parts of the magnetic resonance image that due to the distortion, adding and erasure of the image information cannot faithfully reproduce the actual anatomical structures.

\section{Magnetic Resonance Image Artifacts 2.1 Chemical Shift Artifact of the 1st kind (Type 1)}

Hydrogen ions $\mathrm{H}^{+}$of different molecules are affected by magnetic field in varying extent. Therefore the precession frequency of water differs from that of fat. The relative shift between the peak of water and fat is equal to $3.5 \mathrm{ppm}$. Due to this frequency shift the misregistration of water/fat tissues is observed. Fat/water overlap and fat/water separation is registered. The protons of water and fat positioned on the same place are assigned to the different pixels. The narrower are bandwidths the higher is the chemical shift and the separation of several pixels is observed. At the same time the time of acquisition is increased [1]. Type 1 artifact due to the chemical shift is observed for the standard sequences exclusively along one, the frequency-encoding, direction. For the echo planar imaging (EPI) sequences the bandwidth in the direction of the phase encoding direction is due to the long echo sequence very narrow, therefore the shift between the signal from water and fat is along the phase encoding direction several times higher than that for the standard sequences. To reduce chemical shift artifacts of the 1st kind it is necessary choose the largest bandwidth possible. This reduces the time of acquisition and leads to a small signal-to-noise ratio. For EPI sequences there is a special scheme of data acquisition that does not allow substantially enlarge the bandwidth in the phase encoding direction. Therefore it is necessary eliminate fat spins by the spectral pulse which saturates fat (Fig. 1).

\subsection{Phase Cancellation-Artifact of the 2nd kind (Type 2)}

Type 2 artifact is also caused by the chemical shift between protons of fat and water. In contrast to the Type 1 artifact, Type 2 artifact can be observed only for gradient echo sequences.

As mentioned earlier, the different Larmor fre- quencies of fat in comparison with water causes a phase shift, which varies with the time of highfrequency excitation. If the phase shift is $180^{\circ}$, the signal of volume elements containing both fat and water will be lower because of the partly or completely deleted magnetization components (",opposed phase" condition) [3]. Therefore the dark lines on the border areas between fat and other tissues can be observed (Fig. 2). The artifacts are visible at $1.5 \mathrm{~T}$ and echo time from $2.2 \mathrm{~ms}$ to $6.6 \mathrm{~ms}$. The artifact Type 2 can be suppressed by the adjusting time of echo. At suitably chosen time of echo spins of fat and water are arranged in the same direction and the signals of both are amplified.

The phase cancellation-artifact of the 2 nd kind can be used for diagnostic purposes and the abdominal imaging, where organs and visceral fat form boundaries [4].
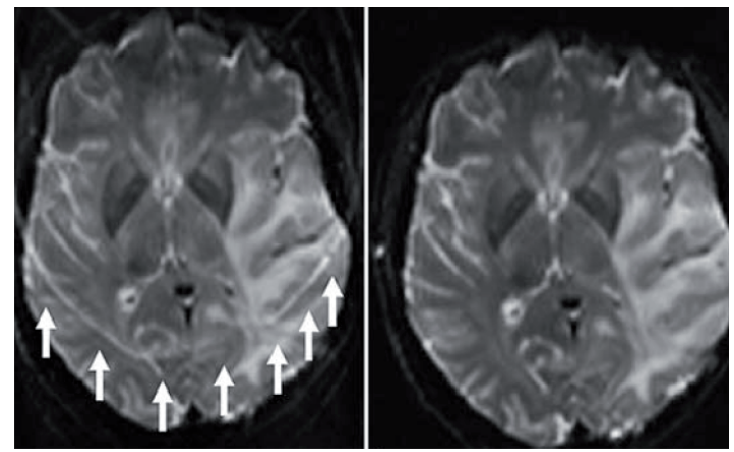

Fig. 1: Spin-Echo-EPl- sequence. Left: Signals from water and fat are shifted to several pixels due to the extremely narrow bandwidth in the phase encoding direction (arrows). Right: Artifacts were eliminated with the aid of the spectral pulse which saturates fat [2].

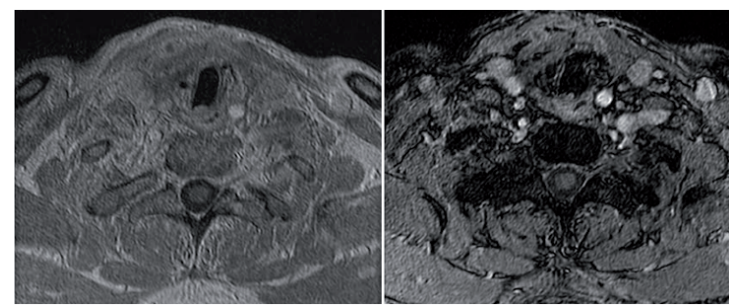

Fig. 2: Phase Cancellation-Artifact of the 2nd kind with gradient echo. Left: time of echo at which protons of water and fat are in phase. Right: the example of image in "opposed-phase" [2].

\subsection{Truncation (Gibb's artifact)}

Truncation is observed as multiple, alternating bright and dark lines (ringing). Obviously it can be seen near tissue borders having abrupt inten- 
sity changes. Sharp border lines cause in k space high amplitudes with high space frequencies. Due to the limited resolution of MR imaging, the image acquisition is always cut from high frequencies. Therefore, there is a periodic fluctuation of intensity at the border (Fig. 3).

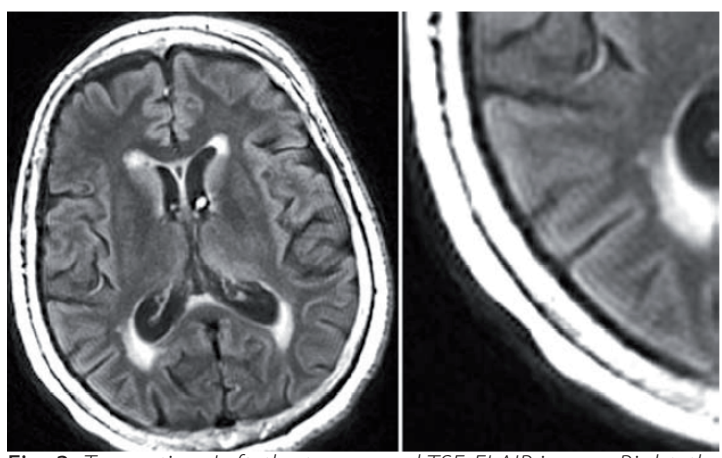

Fig. 3: Truncation. Left: the transversal TSE-FLAIR image. Right: the cut of an image with the signal oscillations ongoing parallel to the edge, the signal of which caused truncation [2].

The observed effect is more pronounced for the lower space resolution [5]. It can be observed when, e.g., EPI sequences are used. The truncation artifact decreases with increased matrix, but does not disappear. In routine operation, the artifact can be suppressed by increasing the spatial resolution, and then it becomes almost undetectable. Increasing of the spatial resolution can be achieved by reducing the size of pixels due to the geometric structure in the image, which can be done by reducing the field of view (FOV) or enlarging the image matrix. Enlarging the image matrix, however, extends the scan time and reduces the signal-to-noise ratio. Alternatively, reducing the FOV can cause aliasing, diagonal lines break due to the limited resolving power. The truncation can be also eliminated by a filter that removes unwanted frequencies [6].

\subsection{Blurring}

If the $\mathrm{k}$ space does not contain high frequencies, the edge and the boundary surfaces of anatomical structures in MR image are unfocused. This is also the case when the number of phase encoding steps is decreased to reduce the acquisition time of dynamic sequences. Even if an adequate number of phase encoding steps were selected, this artifact may occur. For example, if the fast imaging techniques such as turbo spin echo (TSE) or
EPI sequence were used (Fig. 4). Because of a long time of acquisition, the external lines of $k$ space are scanned only with the noise [2].
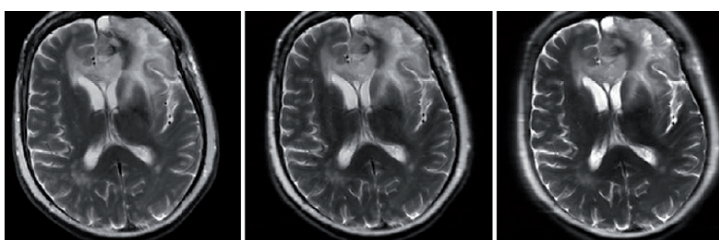

Fig. 4: Transveral TSE images with the turbo-factor of 9, 17 a 31 (from left to right). The higher is the turbo-factor value, the more pronounced is the blurring [2].

Image blurring can be avoided only if the signal from the outer lines of the $k$ space is caught. This can be achieved by selecting a proper scheme of acquisition. Sequential acquisition methods of the k space are better than centric ones: in sequential reading at least half of the high-frequency signal information is recorded at a short time of echo. Another way of the artifact reduction is represented by the shorter acquisition time for the whole sequence of echoes. In doing so, the length of the sequence is reduced, while the acquisition time is extended. The sequence of echoes can also be shortened if the bandwidth is increased, which shortens the time of the acquisition. However, this procedure deteriorates the signal to noise ratio.

\subsection{Crosstalk}

If multislice acquisitions are overlapping, the protons belonging to the more than one slice get saturated. Crosstalk artifact can be prevented by choosing the gap between the layers, representing $10-20 \%$ of the layer thickness, in which no excitation or acquisition is carried out. The shorter is the high-frequency pulse, the larger gap between layers has to be selected. However, in that case there is a risk that small lesions in the gap may be overlooked.

The second method of the artifact removing consists in a procedure where layers are not excited in the order corresponding to the position of the layer, but the odd layers are scanned first and afterwards the even ones. This procedure allows reaching a maximal time delay between the excitation of two adjacent layers. Combination of both methods can almost completely prevent crosstalk artifacts even at very small gaps between the layers (5-10\% of the thickness of the layer). 


\subsection{EPI - ghosting}

When EPI sequences are used, switching and reading of gradients must be performed very quickly allowing a quick scan of k space lines. In doing so, eddy currents are created in the scanner that resist to the gradient structure. That's why the signal maximum is not registered in the middle of the $k$ space but at the odd steps of the phase encoding behind it and at even steps in front of it. Due to the "zigzag" lines of the signal maximum EPI ghosts are generated - the original structure of the body is shifted by half of the FOV in the direction of the phase encoding direction (Fig. 5).

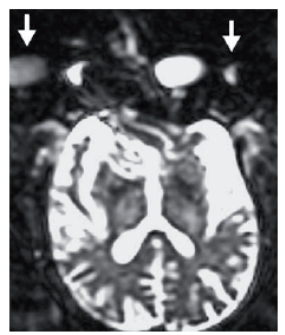

Fig. 5: EPl-ghosting for Spin-Echo-EPI sequences. The displayed object appears as a ghost with the shift of half FOV in the phase encoding direction (from right to left) [2].

The artifact can be avoided by setting the shape of the gradient pulse and the time of switching gradients (preemphase) [7].

While in the past it was possible to perform this adjustment only manually, the new generation of scanners allows the correction of EPI ghosting automatically.

\subsection{EPI - ghosting}

The partial volume artifact occurs when the spatial resolution is so small that several entities of the tissue within the volume element (voxel) are found. In this voxel the signal intensity is indicated as a weighted average of various intensities. The partial volume artifact occurs in 2D sequences mainly in the direction of the layer because of a large anisotropy of spatial resolution of these sequences. In general, a partial volume effects lead to the fact that the small and the low contrast structures cannot be distinguished. During the volumetric examination the partial volume effect leads to the underestimation of the lesion volume. In addition, especially when the sequences of gradient echoes are applied, these structures pretend that they are not present. The partial volume artifact can be minimized by increasing the spatial resolution. When 2D sequences are used, an attention must be given to the thickness of the layer. However, this procedure reduces the signal-to-noise ratio. For T2-weighted sequences, particularly for EPI sequences, and for the gradient echo sequences as well, the reduction of the signal-to-noise ratio paradoxically leads to the smaller values than it can be expected from the reduced voxel. The reason is that an un-phasing inside the voxel, and the associated reduction of the signal, is smaller for the smaller voxels [8].

\subsection{Saturation artifacts}

When high-frequency pulses with a high repetition rate are used, the longitudinal magnetization cannot be sufficiently restored by $\mathrm{T1}$ relaxation between pulses. It can result in the fact that the effective repetition time is shorter than the repetition time set for the sequence. If layers or sets of layers overlap, saturation artifacts can blocks occur in overlapping areas. During the time of flight (TOF) MR angiography examination, saturation artifacts due to the very short times of repetition for suppressing static tissue are very common. The saturation artifacts in MR-TOF angiography can be avoided if a block of layers is placed perpendicularly to the blood vessels. If a larger volume of blood vessels is to be displayed, it is appropriate to choose several overlapping blocks of layers instead of one thick block [9].

TONE technique represents another method of reducing the saturation artifacts in the thick layers or in case of the slow flow. In this method, a tilt angle of the high frequency pulse is increased linearly from the proximal end to the distal end of the 3D block, which ensures a uniform contrast between the blood vessel and the background (tissue) [10].

\section{Conclusions}

In this review the main types of MR artifacts due to the examination method and the scanning sequences are presented. The characteristic properties and the physical and the technical preconditions of given artifacts are given.

\section{Acknowledgments}

This study was supported by the grants VEGA 1/0515/13 (25\%) of the Ministry of education of 
the Slovak Republic and partially by the Agency of the Slovak Ministry of Education for the Structural Funds of the EU under projects: ITMS: 26220220163 (25\%), ITMS: 26220220143 (25\%), ITMS: 26220120058 (25\%).

\section{References}

[1] Smith, A.S., Weinstein, M.A., Hurst, G.C., DeRemer, D.R., Cole, R.A., Duchesneau, P.M. (1990). Intracranial chemical shift artifacts on MR images of the brain: observations and relation to sampling bandwidth. Am J Roentgenol, 154, 6, 1275-1283.

[2] Heiland, S. (2009). Detect and avoid artifacts. Radiologie up2date, 9, 4, 303-318.

[3] Savci, G., Yazici, Z., Sahin, N., Akgöz, S., Tuncel, E. (2006). Value of chemical shift subtraction MRI in characterization of adrenal masses. Am J Roentgenol, 186, 1, 130-135.

[4] Venkataraman, S., Braga, L., Semelka, R. (2002). Imaging the fatty liver. Magn Reson Imaging C, 10, 1, 93-103.

[5] Czervionke, L.F., Czervionke, J.M., Daniels, D.L., Haughton, V.M. (1988). Characteristic features of MR truncation artifacts. Am J Roentgenol, 151, 9, 815-824

[6] Runge, V.M., Nitz, W.R., Schmeets, S.H. (2009). The Physics of Clinical MRI Taught Through Images. Thieme, New York.

[7] Papadakis, N.G., Martine, K.M., Pickard, J.D., Hall, L.D., Carpenter, T. A., Huang, C.L. (2000). Gradient preempasis calibration in diffusion-weighted echo-planar imaging. Magn Reson Med, 44, 4, 616-624. 